\title{
Firefly Optimization Algorithm for the Prediction of Uplift due to High-Pressure Jet Grouting
}

\author{
Wukui Dai $\mathbb{D}^{1,2}$ Li Liang $\mathbb{D}^{1}{ }^{1}$ and Bingji Zhang $\mathbb{D}^{2}$ \\ ${ }^{1}$ School of Resources \& Civil Engineering, Northeastern University, Shenyang 110819, China \\ ${ }^{2}$ China Northeast Architectural Design \& Research Institute Co. Ltd, Shenyang 110006, China \\ Correspondence should be addressed to Wukui Dai; dwk106@126.com
}

Received 23 June 2020; Revised 30 October 2020; Accepted 25 November 2020; Published 10 December 2020

Academic Editor: Weerachart Tangchirapat

Copyright (c) 2020 Wukui Dai et al. This is an open access article distributed under the Creative Commons Attribution License, which permits unrestricted use, distribution, and reproduction in any medium, provided the original work is properly cited.

A project that needs to be uplifted by high-pressure jet grouting (HPJG) is exposed to particular geological and engineering circumstances; meanwhile, HPJG has intense subjectivity, short of the theoretical base, to ascertain the influence angle $\beta$ and enlarged radius $\Delta a$, which are the main parameters that affect the uplift effect. Therefore, we proposed a new method based on the firefly optimization algorithm to search for the optimal solution for the target function. Stochastic medium theory (SMT) was used in this article, in which the effect of single-pile HPJG was simulated as the superposition effect of the foam slurry at the same distance, to construct a stochastic medium prediction model of the effect of uplift due to multi-HPJG. In accordance with the range of the prediction results of single-pile HPJG and combined with in situ monitoring data to define the target function, the optimal parameters are substituted into the prediction model to predict the subsequent uplift effect due to HPJG. As a result of the global optimization capacity and by comparison with the genetic algorithm, the FOA has a greater advantage in terms of effectiveness and precision. Finally, it is proven that the prediction result meets the requirement of the prediction in advance by statistical data.

\section{Introduction}

HPJG construction technology was developed in Japan in the 1970 s and has been used as a primary technique in ground reinforcement projects because it has a wide range of applications in small working spaces; however, piles provide a higher bearing capacity [1-5].

Grouting uplift technology reinforces the target ground by the grouting method and forces the ground surface or building to move upward to control and rectify the subsoil subsidence of the existing building. Traditional grouting technology mainly reinforces subsoil; however, the uplift effect has been focused on and gradually used since the 1980s. In terms of theoretical research, Maag studied the grouting theory in 1938 in granular material assuming slurry scatters in a sphere shape and established an equation relating the factors of the grouting pressure, slurry volume, scatter radius, void ratio, and permeability. For the first time in 1969, Graf proposed that the uplift effect of consolidation grouting can be used to rectify buildings and described the working process of grouting slurry
[6]. Graf proposed that consolidation grouting may improve and consolidate loose subsoil. Furthermore, Graf proposed that buildings with the differential settlement can be rectified by grouting bodies and uplift effects. Graf pointed out that the slurry was grouted by consecutive pressure into the subsoil, and the subsoil beyond the slurry foam would be destroyed in a cone mode when the pressure was sufficiently high [7]. Greenwood compared consolidation grouting with other grouting modes in 1987, focusing on the relation between a slurry with a different consistency and replacement mode in the grouting position [8]. Sagaseta derived displacement solutions via Mirror Theory induced by strata loss or grouting within a finite depth in an elastic infinite space $[9,10]$. Gollegger studied the solutions of the displacement field induced by compensation grouting based on Sagaseta's results [11]. Komiya et al. studied the influence law of strata displacement control based on the grouting uplift effect on tunnel digging in soft clayey soil with in situ and laboratory tests in Tokyo in 2001. EI-Kelesh et al. proposed the theory model of consolidation grouting based on the observed case of subsoil displacement by Brown Warner, and Graf et al. 
proposed the theoretical design methods of consolidation grouting [12-14]. In 2012, combined with existing projects, and by determining whether the uplift process came to the increment of the subsoil volume by volume increment of the unit subsoil, Zhang Min et al. proposed a method of simulation by the numerical computation in the uplift process induced by consolidation grouting [15].

HPJG is widely applied to ground reinforcement and rectification projects; however, there is no better way to quantitatively predict the uplift effect of HPJG, mainly because it depends on the following monitoring data and experience to control the construction process. Thus, producing effective uplift schemes according to existing projects and reasonable predictions about uplift is substantially important.

With an existing project, based on the stochastic medium theory in rock movement introduced by the Polish scholar Litwiniszyn [16-20], this article established a theoretical computation model of simulating the uplift process induced by HPJG. Modeling is combined with optimization, but as for the complex matter of engineering and scientific optimization which cannot be solved by traditional optimization methods, the swarm intelligence optimization algorithm drew attention gradually. By simulating the group behavior of all kinds of natural social creatures, and utilizing the information interchange and cooperation among the individuals to achieve the purpose of optimization, people put forward the swarm intelligence optimization algorithm. Compared with other optimization methods, it has the advantage of simplicity and high effectiveness, it has been applied to the fields of function optimization, combinatorial optimization, artificial life, automatic control, and machine learning. Combined with the in situ monitoring data, this article used swarm intelligence optimization algorithm and genetic algorithm of evolution algorithm to define target function, respectively, in order to obtain the main flurry foam influence angle $\beta$ and enlarged radius $\Delta a$, which are the main parameters that affect the uplift effect induced by HPJG, and then substitute the optimal parameter into the prediction model, respectively, to predict the uplift displacement due to HPJG theoretically. Meanwhile, we made a comparison with the two kinds of optimization algorithms in many ways and made a mutual check with the in situ monitoring data. The purpose was to control and rectify the foundation subsidence of existing buildings and structures based on this method.

\section{The Surface Uplift Induced by HPJG}

2.1. The Uplift Mechanism of HPJG. The mechanism of HPJG comprised five effects: the effect caused by HPJG through cutting the soil, mixed stirring, an uplift and replacement effect, backfilling and soil compaction, and infiltration consolidation. As HPJG is different from conventional pressure grouting, it was able to rapidly fill the grooves generated through flushing and the gaps between the soil particles by using a high-pressure cement slurry. The jetting effect on the soil surface tended to produce two types of forces: on the one hand, the jetting effect on the zone that was the nearest to the nozzle was exerted on a very small surface, and the pressure was far larger than the self-weight stress of the soil; on the other hand, the zone was further from the nozzle, and therefore, the jet pressure was unable to damage the soil; however, the soil was compacted, and some of the liquid jets was squeezed into the soil to produce an extrusion pressure. The effect on surface uplift mainly involved the effect of filling and compaction. HPJG has favorable pile-forming performance, and the soil was compressed via the thick slurry injection in a borehole to realize surface uplift, which was different from pressure grouting. This method had a smaller dispersion area, and the effect of single piles on the surface uplift was less obvious than that of pressure grouting; hence, the superposition effect of multi-HPJG grouting comprising multiple jet grouting piles can effectively realize the purpose of rectification [2-5].

2.2. The SMT Model of the Surface Uplift Effect of HPJG. Based on SMT, the vertical uplift displacement of a single foam slurry compared to the point $(x, y, z)$ could be equivalent to the total influence of the infinite expansion of infinitesimal voids containing slurry (Figure 1) [21-26].

The uplift displacement was

$$
W=\frac{1}{r^{2}(z)} \exp \left[\frac{-\pi}{r^{2}(z)}\left(x^{2}+y^{2}\right)\right] \mathrm{d} \xi \mathrm{d} \zeta \mathrm{d} \eta,
$$

where $r(z)$ refers to the radius of the main influence on the element excavated at level $z$, which is also a function related to $z$. Hence, it is considered to have a linear relationship with $z$, namely, $r(z)=z / \tan \beta$, where $\beta$ denotes the main influence angle of the upper soil on the foam slurry. Its value depends on the strata conditions in the region subjected to grouting slurry treatment.

The uplift of the subsoil is due to the enlargement of the foam slurry. It was assumed that the initial volume of the foam slurry before grouting was $\Omega$, which increased to $\omega$ after grouting. Therefore, the surface uplift of a single foam slurry should be the difference between the surface uplift induced by the volume $\Omega$ of the foam slurry before grouting and the volume of the foam slurry after grouting. The uplift at $(x, y, z)$ in the soil was

$$
\begin{aligned}
W(x, y, z)= & \iiint_{\Omega} \frac{\tan ^{2} \beta}{(z-\eta)^{2}} \exp \left\{\frac{-\pi \cdot \tan ^{2} \beta}{(z-\eta)^{2}}\left[(x-\xi)^{2}+(y-\zeta)^{2}\right]\right\}\left\{\frac{-\pi \cdot \tan ^{2} \beta}{(z-\eta)^{2}}\left[(x-\xi)^{2}+(y-\zeta)^{2}\right]\right\} \mathrm{d} \xi \mathrm{d} \zeta \mathrm{d} \eta \\
& -\iiint_{\omega} \frac{\tan ^{2} \beta}{(z-\eta)^{2}} \exp \left\{\frac{-\pi \cdot \tan ^{2} \beta}{(z-\eta)^{2}}\left[(x-\xi)^{2}+(y-\zeta)^{2}\right]\right\} \mathrm{d} \xi \mathrm{d} \zeta \mathrm{d} \eta
\end{aligned}
$$




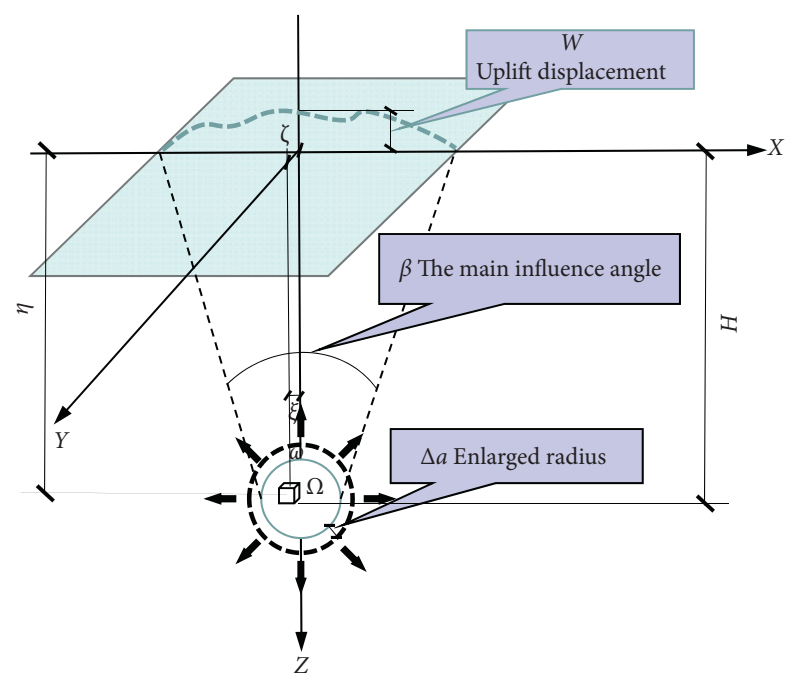

FIgURE 1: Schematic diagram of the elements of the foam slurry analyzed based on SMT.

Assuming that the foam slurry has a spherical shape, the foam slurry is symmetrically enlarged, and the radius $\Delta a$ and the uplift at the surface $(x, y, 0)$ can be determined via

$$
\begin{aligned}
W(x, y, 0)= & \int_{a_{1}}^{b_{1}} \int_{c_{1}}^{d_{1}} \int_{e_{1}}^{f_{1}} \frac{\tan ^{2} \beta}{\eta^{2}} \exp \left\{\frac{-\pi \cdot \tan ^{2} \beta}{\eta^{2}}\left[(x-\xi)^{2}+(y-\zeta)^{2}\right]\right\} \mathrm{d} \xi \mathrm{d} \zeta \mathrm{d} \eta \\
& -\int_{a_{2}}^{b_{2}} \int_{c_{2}}^{d_{2}} \int_{e_{2}}^{f_{2}} \frac{\tan ^{2} \beta}{\eta^{2}} \exp \left\{\frac{-\pi \cdot \tan ^{2} \beta}{\eta^{2}}\left[(x-\xi)^{2}+(y-\zeta)^{2}\right]\right\} \mathrm{d} \xi \mathrm{d} \zeta \mathrm{d} \eta
\end{aligned}
$$

Here,

$$
\begin{aligned}
& a_{1}=H-a, \\
& b_{1}=H+a, \\
& c_{1}=-\sqrt{a^{2}-(H-\eta)^{2}}, \\
& d_{1}=\sqrt{a^{2}-(H-\eta)^{2}} \\
& e_{1}=-\sqrt{a^{2}-(H-\eta)^{2}-\zeta^{2}}, \\
& f_{1}=\sqrt{a^{2}-(H-\eta)^{2}-\zeta^{2}} \\
& a_{2}=H-(a+\Delta A) a \\
& b_{2}=H+(a+\Delta a), \\
& c_{2}=-\sqrt{(a+\Delta a)^{2}-(H-\eta)^{2}}, \\
& d_{2}=\sqrt{(a+\Delta a)^{2}-(H-\eta)^{2}}, \\
& e_{2}=-\sqrt{(a+\Delta a)^{2}-(H-\eta)^{2}-\zeta^{2}}, \\
& f_{2}=\sqrt{(a+\Delta a)^{2}-(H-\eta)^{2}-\zeta^{2}},
\end{aligned}
$$

where $a$ represents the initial radius of the foam slurry and $\Delta a$ refers to the enlargement of the radius of the section.

As shown in Figure 2, the effect of single-pile HPJG was simulated as the superposition effect of foam slurry at the same distance. Meanwhile, superposition theory can be applied to multi-HPJG to calculate the surface displacement, and the uplift effect of multi-HPJG on the surface soil via equation (5) is

$$
W(x, y, 0)=\sum_{k=1}^{l} \sum_{j=1}^{m} \sum_{i=1}^{n}\left\{\begin{array}{l}
\int_{a_{1}}^{b_{1}} \int_{c_{1}}^{d_{1}} \int_{e_{1}}^{f_{1}} \frac{\tan ^{2} \beta}{\eta^{2}} \exp \left\{\frac{-\pi \cdot \tan ^{2} \beta}{\eta^{2}}\left[(x-\xi)^{2}+(y-\zeta)^{2}\right]\right\} \mathrm{d} \xi \mathrm{d} \zeta \mathrm{d} \eta- \\
\int_{a_{2}}^{b_{2}} \int_{c_{2}}^{d_{2}} \int_{e_{2}}^{f_{2}} \frac{\tan ^{2} \beta}{\eta^{2}} \exp \left\{\frac{-\pi \cdot \tan ^{2} \beta}{\eta^{2}}\left[(x-\xi)^{2}+(y-\zeta)^{2}\right]\right\} \mathrm{d} \xi \mathrm{d} \zeta \mathrm{d} \eta
\end{array}\right\} .
$$




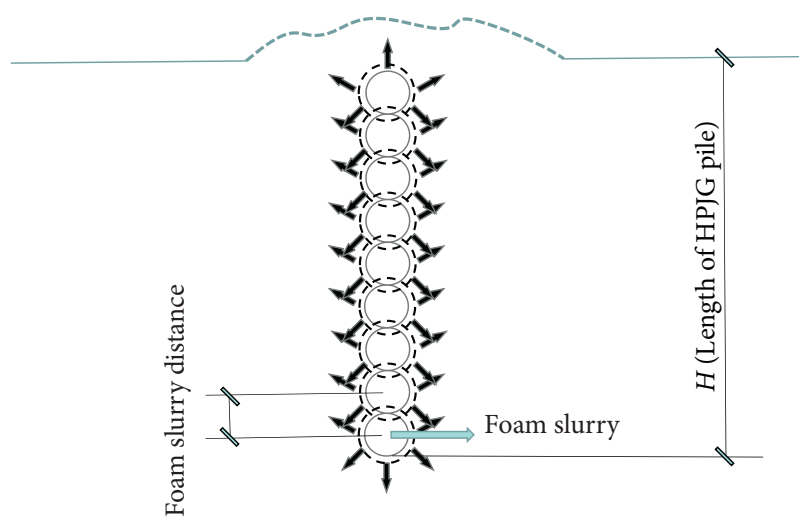

FIGURE 2: The superposition simulation of the foam slurry during HPJG.

Here, $n$ refers to the number of foam slurry cells that formed a single HPJG pile, $m$ is the number of HPJG piles in the $x$-direction, and lis the number of HPJG piles in the $y$-direction.

\section{The Scope of the Prediction of Uplift due to HPJG}

3.1. The Uplift Case. The wastewater treatment plant of a petroleum refinery was taken as the research subject. The foundation soil of the plant was backfilled. The backfill soil had a thickness ranging between 9 and $17 \mathrm{~m}$ and mainly contained red clay. Dynamic compaction was completed in 2013, and the main work commenced in the second half of 2013. As the drainage system of the wastewater treatment plant had not yet developed, the foundation soil was immersed in water; therefore, the quality and uniformity of the foundation after dynamic compaction were influenced. Since October 2014, the constructed biochemical pond showed uneven subsidence and tensile damage, which affected its normal use (Figure 3). The reinforced and uplifted biochemical pond is taken as an example.

The biochemical pond had a raft foundation with a thickness of $600 \mathrm{~mm}$ (Figure 4). The pond was divided into six blocks by two east-west temperature joints and one south-north temperature joint. The settlement between 39 and $168 \mathrm{~mm}$ occurred by December 9, 2015. The maximum differential settlement was $70 \mathrm{~mm}$; taking the southwest block of the biochemical pond as an example, the location with the maximum settlement was in the northeast (namely, the intersection zone of the temperature joints). Leveling treatment was required to be conducted on each block of the pond considering its subsequent use. By taking the enforceability of the consolidation and rectification for the structure of the pond into account, an HPJG scheme was finally chosen.

The basic parameters of the subsoil of the biochemical pond are shown in Table 1. The subsoil is backfilled after dynamic compaction, which can be seen as a stochastic medium; thus, the stochastic medium is suitable for predicting the surface movement and deformation due to HPJG construction. After the in situ pile test, the parameters shown in Table 2 are taken as those for HPJG construction. The results of the in situ pile test are shown in Figure 5.

3.2. Predicted Scope Affected by a Single HPJG. The parameters of pressure grouting without considering the pressure of the buildings/structures consisted of the main influence angle $\beta$ and enlarged radius $\Delta a$ for the section of a single foam slurry, which were $50^{\circ}$ and $0.1 \mathrm{~m}$, respectively. The initial radius of the foam slurry was $0.025 \mathrm{~m}$, the distance of the foam slurry with the vertical displacement of a single pile was $0.6 \mathrm{~m}$, and the length of the HPJG pile was set to $15 \mathrm{~m}$. The vertical displacement of the single-grouting pile to the surface was calculated (Figure 6).

As shown in the figure, the calculated results of $\beta$ and $\Delta a$ were slightly greater than those of the tested piles. This was due to the rapid hoisting of the jet shaft, water-cement ratio of the slurry, and grouting pressure being large, while the slurry was dispersed over a small range. Unlike the pressure grouting technique that keeps pumping thick slurry into the ground surface, the volume of slurry columns increased to produce a greater uplift pressure, which, therefore, led to a larger displacement and surface uplift. According to the calculated results, the surface uplift induced by a singlegrouting pile of HPJG was mainly within $2 \mathrm{~m}$ around the grouting borehole, the most concentrated displacement was found in the center of the grouting borehole, and the effect on the displacement of the stratum $7 \mathrm{~m}$ from the grouting borehole tended to zero.

The aforementioned equation and analysis showed that the main factors affecting surface uplift were the influence angle $\beta$ and enlarged radius of the section for the upper soil and foam slurry under an assumed distance of foam slurry dissipation. Since they are comprehensive parameters and take into consideration the pressure from the superstructure and relevant constraints, parameter selection via geological analysis alone was apt to be subjective; hence, the determination of equivalent $\beta$ and $\Delta a$ values under specific conditions was essential to calculate the uplift of HPJG on existing buildings/structures.

By integrating the calculated results, the layout of the jet grouting pile scheme for consolidating and rectifying this pond is shown in Figure 7. The construction of the jet grouting pile was implemented in the order indicated by the arrows. For different measurement points on the bottom slab, it was considered that the range of influence of the jet grouting pile that contributed to their uplift was within approximately $7.5 \mathrm{~m}$ of each measurement point.

\section{The Comparison of the Optimization Algorithms for $\beta$ and $\Delta a$}

In practice, traditional algorithms have experienced great challenges due to optimization issues. More swarm intelligence algorithms have been proposed. In recent decades, scholars have proposed a series of swarm intelligence optimization algorithms to simulate the habits of populations of animals by simulating the social behaviors of insects and animals. It is essential to search for an 

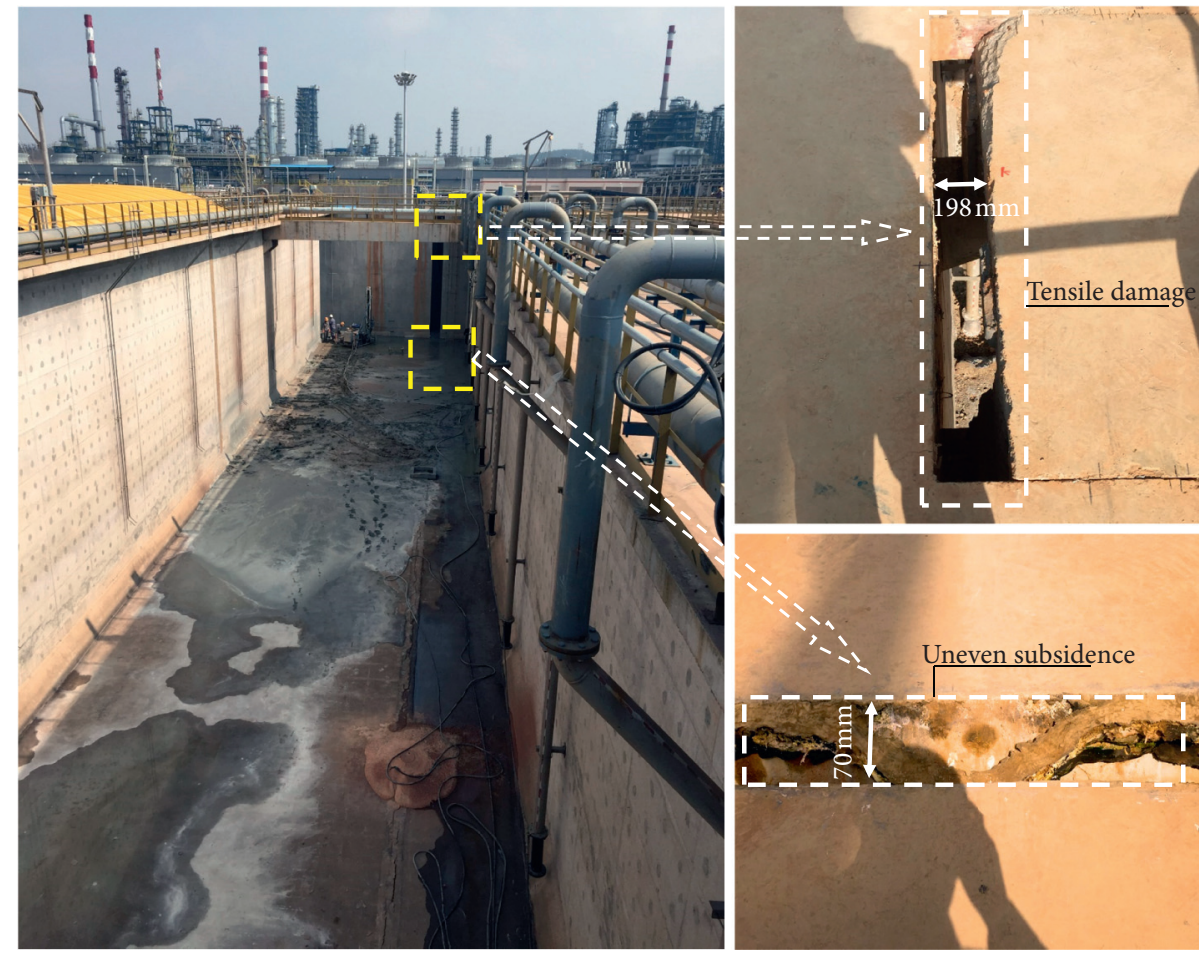

FIgURE 3: Tensile damage and uneven subsidence of the biochemical pond.

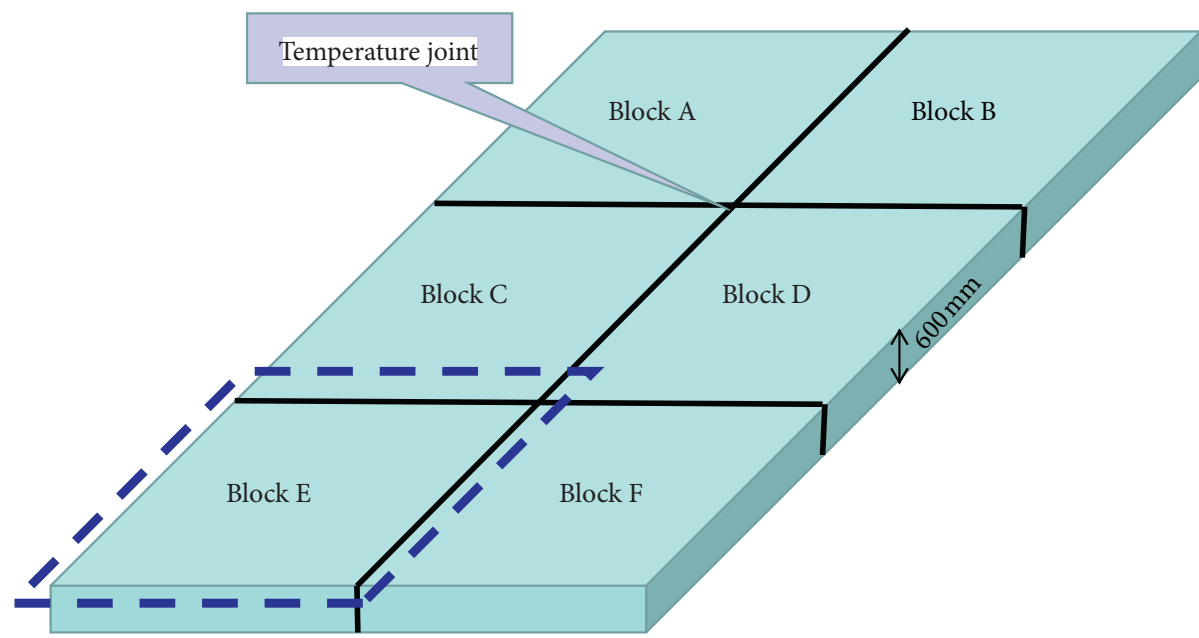

FIgURE 4: A base slab of the biochemical pond.

TABLE 1: The characteristic value of the bearing capacity of the subsoil and compression modulus.

\begin{tabular}{lcc}
\hline Soil classification & Characteristic value of the bearing capacity $(\mathrm{kPa})$ & Compression modulus (MPa) \\
\hline (1) Plain fill & 160 & 6.4 \\
(1) Plain fill & 60 & 3 \\
(2) Silty clay & 140 & 4.3 \\
(2) Silty clay & 100 & 3.9 \\
(3) Clay & 180 & 6.4 \\
(4) Clay & 120 & 5 \\
\hline
\end{tabular}

algorithm for optimization issues. The typical intelligence algorithms are genetic algorithm, ant colony algorithm, particle swarm algorithm, bee colony algorithm, and firefly optimization algorithm [27-29]. Ant colony algorithm has a strong ability to find a better solution, but it takes a long time to search when the group size is big. The particle 
TABle 2: The parameters of the test piles.

\begin{tabular}{lccccccc}
\hline $\begin{array}{l}\text { Drilling } \\
\text { depth }(\mathrm{M})\end{array}$ & $\begin{array}{c}\text { Ratio of water } \\
\text { to cement }\end{array}$ & $\begin{array}{c}\text { Grouting } \\
\text { pressure }(\mathrm{MPa})\end{array}$ & $\begin{array}{c}\text { Hoisting velocity } \\
(\mathrm{cm} / \mathrm{min})\end{array}$ & $\begin{array}{c}\text { Rotatory } \\
\text { velocity }(\mathrm{r} / \mathrm{min})\end{array}$ & $\begin{array}{c}\text { Wind pressure } \\
(\mathrm{MPa})\end{array}$ & $\begin{array}{c}\text { Cement } \\
\text { amount }(\mathrm{kg} / \mathrm{m})\end{array}$ & $\begin{array}{c}\text { Pile diameter } \\
(\mathrm{mm})\end{array}$ \\
\hline 15 & $1: 1$ & 28 & 12 & 15 & 0.6 & 288 & $>600$ \\
\hline
\end{tabular}

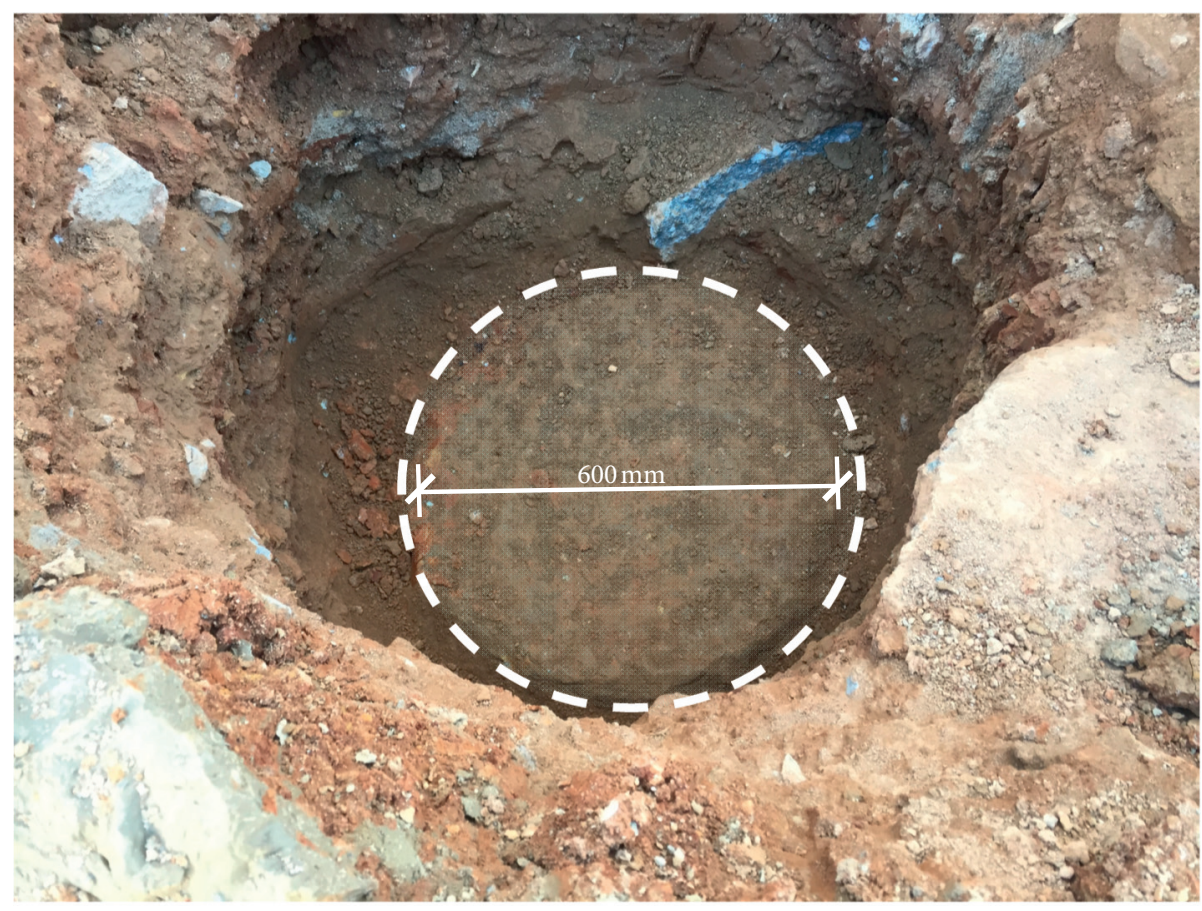

Figure 5: Diameter of the in situ test pile.

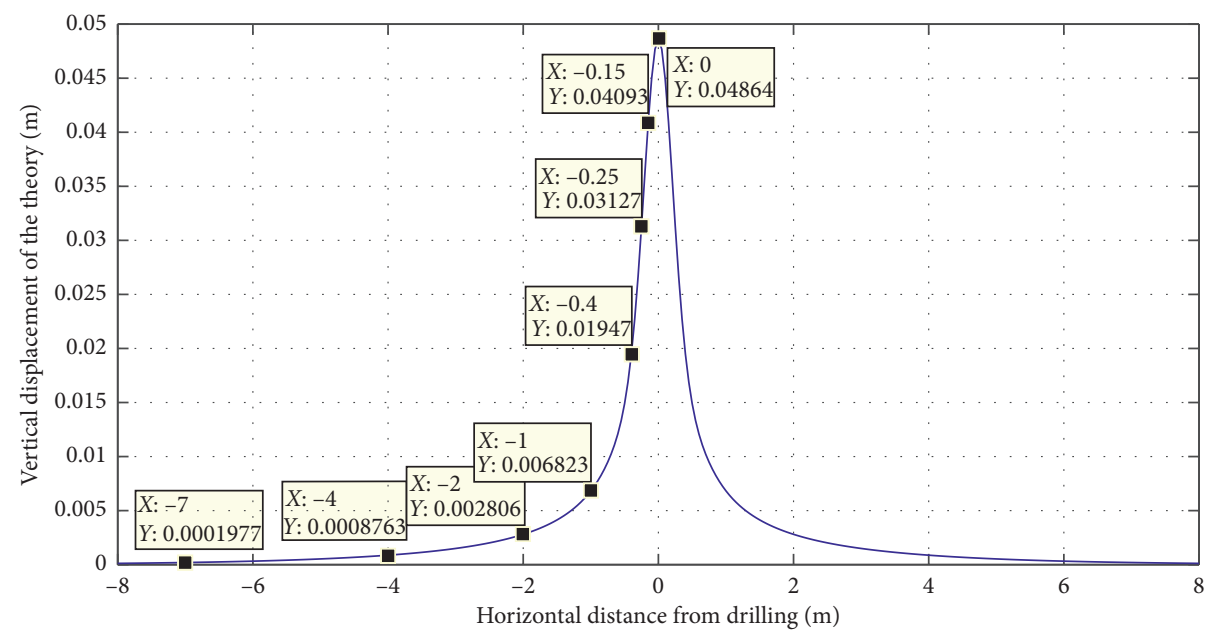

FIGURE 6: The calculated vertical displacement of the ground surface after conducting a single HPJG according to provisional parameters.

swarm algorithm has a simple and easy procedure to achieve the purpose with fewer parameters, but when the self-information and individual information are in the dominant position, it falls to local optimization easily. Artificial bee colony algorithm has many advantages such as discrete problem solution, but as for the global search in complex space, it increases the time complexity inevitably such that a lot of time is consumed. Among these algorithms, both the genetic algorithm and the FOA have better convergence with less runtime and high robustness. Moreover, the two algorithms have an excellent global search capacity compared with other algorithms. In this article, the FOA and a genetic algorithm were applied to search the parameters of the influence angle $\beta$ and enlarged 


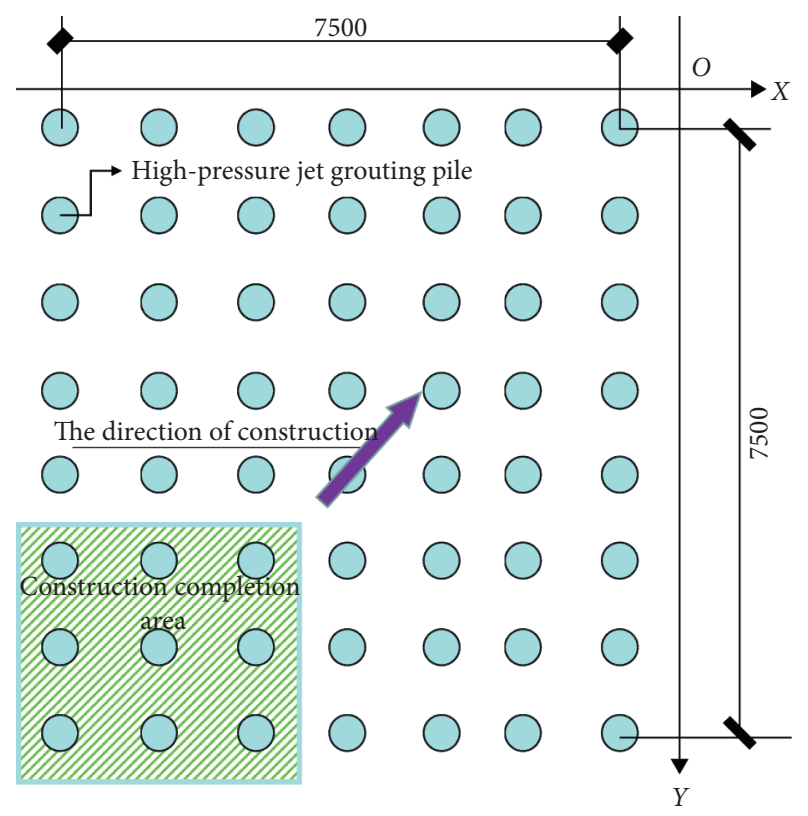

FIGURE 7: HPJG layout within the E slab of the biochemical pond.

radius $\Delta a$, which affect the ground uplift. In addition, we compared the complexity, program running times, and prediction precision of the two algorithms to obtain a better theoretical method of uplift prediction due to HPJG [30-35].

4.1. Optimization of the Parameters Based on a Genetic Algorithm. The theory of genetic algorithms sourced from the evolutionary process of genetics and selection in nature and its structure is illustrated in Figure 8 [36-38].

According to the construction process used in this project, the displacement change $W_{0}$ at early stages which were used to measure the displacement of the prediction points at the later stages of construction of the jet grouting pile and the uplift displacement $W$ came from the integrating equation (6). The uplift displacement results $W$ were obtained and defined as the target function $T(x)$ :

$$
T(x)=\frac{1}{\left|W-W_{0}\right|+1} .
$$

Given that $x=\left\{\tan ^{2} \beta, \Delta a\right\}$, a group of parameters $x$ is sought to maximize the value of the target function, which in fact involves an optimal issue to make the target function satisfy the given demands.

Using a genetic algorithm, the population is set to $n=40$. By combining our experience with pressure grouting, taking the upper limits of $\beta=50^{\circ}$ and $\Delta a=0.5 \mathrm{~m}$ and the lower limits of $\beta=0^{\circ}$ and $\Delta a=0 \mathrm{~m}$, the crossover probability, mutation probability, and calculation precision are set to be $0.9,0.01$, and 0.01 , respectively.

By taking the intersection of the two movement joints on plate $E$ as an example, the uplift at point $O$ was $3.3 \mathrm{~mm}$ when the jet grouting pile was set as shown in the shaded zone in Figure 8 . This value was substituted into the target function to search for optimized parameters $\tan ^{2} \beta$ and $\Delta a$.

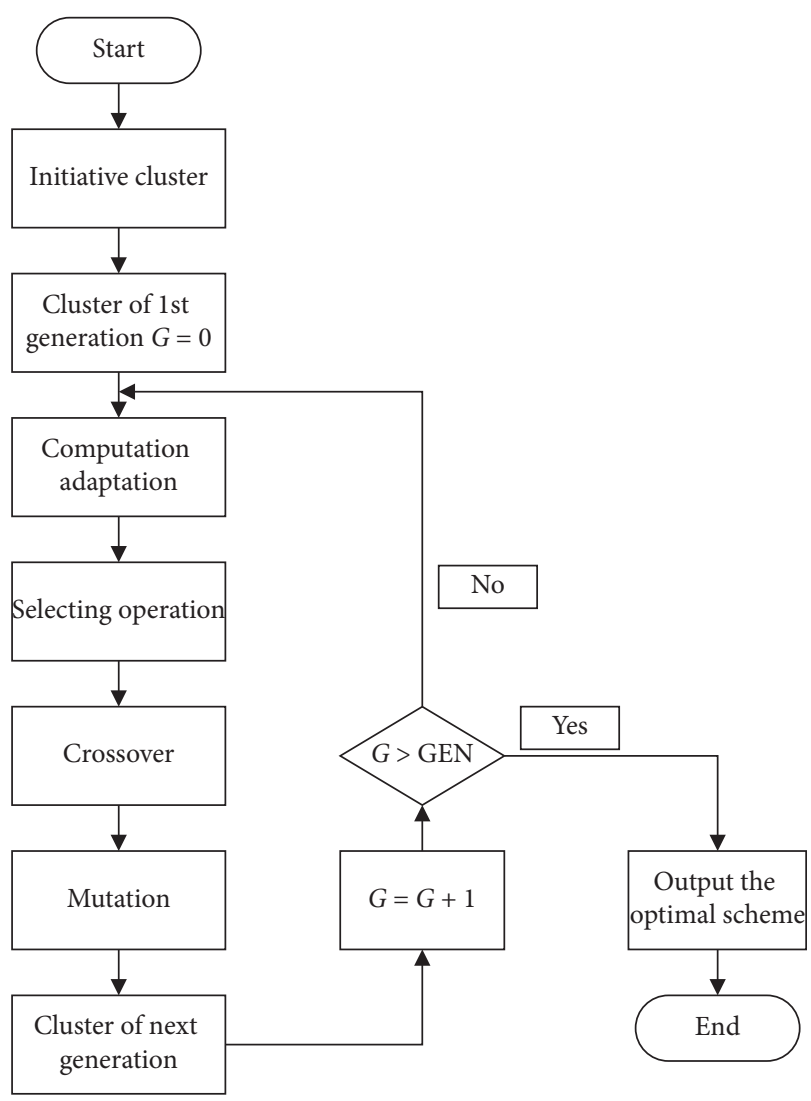

Figure 8: The structure of a genetic algorithm.

The selection, crossover, and mutation are stochastic operations rather than having certain precise regularity. Stochastic methods are mainly used to search for the optimal solution, select the individual through the selection probability by the roulette wheel selection method, and approach the optimal solution. Crossover embodies the emergence of an optimal solution; mutation embodies the overlap of the overall optimal solution. The program is composed to perform the genetic optimization computation. Since the core algorithm of the genetic algorithm contains the stochastic searching method, the optimal solution of one running time has some difference from the actual optimal solution. Thus, we calculated the statistics of the optimal resolution target function of 25 running times, and the optimal result of the target function ranged between 0.9986 and 0.9997. $\tan ^{2} \beta=1.0692\left(\beta=45.96^{\circ}\right)$ and $\Delta a=0.2222 \mathrm{~m}$ corresponding to the predicted optimal result of 0.9997 were used as influencing parameters for the uplift of HPJG.

4.2. Optimization Based on FOA. The FOA evolved from the firefly population that, in nature, communicates information by means of luminescence, as first proposed by Yang in 2009. It is a novel bionic swarm intelligence optimization algorithm. By simulating the behavior of fireflies, a stochastic optimization algorithm is constructed: its principle involves the use of points in a search space to simulate individual fireflies. The searching and optimizing process in the FOA is simulated as the process used to govern the attraction and 
movement of fireflies. The target function of solving problems is to solve the superiority and inferiority of firefly individuals. The process of the survival of the fittest was analogous to this optimization problem. As the FOA is easy to use, it exhibits greater capabilities and faster convergence when searching for a globally optimal solution compared to genetic algorithms [39-43].

4.2.1. The Determination of Target Functions. According to the monitoring result for $W_{0}$ on the piles within the shadow in Figure 7 and the calculated prediction of the uplift effect displacement result $W$ via equation (5), the target function $F(x)$ is

$$
F(x)=\left|W-W_{0}\right|
$$

Similarly, a group of parameters $x$ is determined, which minimizes the value of the target function. Optimized values can then be used to predict the uplift at a later stage.

4.2.2. The Mathematical Description and Analysis of FOA. The luminance and degree of attraction are two essential factors in FOA: the luminance reflects the superiority and inferiority for the location of fireflies and determines the direction of movement of the fireflies, while the degree of attraction determines the distance moved. By constantly updating the luminance, the optimized target is reached.

The relative luminance of the fireflies is expressed as

$$
I=I_{0} \times e^{-\gamma r_{i j}}
$$

where $I_{0}$ is the maximum luminance of fireflies, which is the luminance at $(r=0), \gamma$ is the coefficient of the light absorption intensity, which mainly indicates that the luminance gradually weakens with increasing distance and adsorption in the propagating medium, and $r_{i j}$ is the distance between $i$ and $j$.

The degree of attraction is given by

$$
\psi=\psi_{0} \times e^{-\gamma r_{i j}^{2}}
$$

where $\psi_{0}$ refers to the maximum degree of attraction, namely, that of light sources $(r=0)$.

When firefly $i$ is attracted by firefly $j$ which emits brighter light, the location to which firefly $i$ is attracted by $j$ is updated as follows:

$$
x_{i}=x_{i}+\psi \times\left(x_{j}-x_{i}\right)+\alpha \times\left(\operatorname{rand}-\frac{1}{2}\right),
$$

where $x_{i}$ and $x_{j}$ are the positions where fireflies $i$ and $j$ are located, $\alpha$ is the movement step size, and rand is a random number on the interval $[0,1]$.

The process of the FOA is demonstrated in Figure 9.

Using the aforementioned algorithm, in the course of optimization and based on the fixed scale of the firefly population, we obtained a series of optimal combinations by the enumeration method for the step size factor and absorption factor of illumination; we then made an analysis comparison with the prediction result and in situ monitoring data to obtain the recommended values for the algorithm. The parameters were set as follows: the scale of the firefly population was $n=15$, the maximum number of iterations was 30, the light absorption intensity was $\gamma=1.0$, the step size factor was $\alpha=0.2$, the search range of $\beta$ was 0 to $50^{\circ}$, and $\Delta a$ was between 0 and $0.5 \mathrm{~m}$.

By optimizing the fireflies using the aforementioned routine in MATLAB $^{\mathrm{TM}}$ to make excitation for the fireflies at an optimal position based on the FOA rules, the computation can be performed until it meets the maximum search times according to the updated spatial position of the fireflies. The optimized results are listed in Table 3.

As shown, the minimum optimal values of the target function were zero in each optimization operation using the FOA: $\tan ^{2} \beta=0.5179\left(\beta=35.74^{\circ}\right)$ and $\Delta a=0.1667 \mathrm{~m}$ were used as the influence parameters of the uplift effect of HPJG in this project.

\section{Analysis of the Uplift Effect Predictions}

5.1. Analysis of Prediction Results. According to the FOA results, taking $1.5 \mathrm{~m}$ and $1.25 \mathrm{~m}$ into account, the author performed tentative computations on the HPJG distance for the pile group within $7.5 \mathrm{~m}$ to the uplift corner point. The prediction results were $44.0 \mathrm{~mm}$ and $61.8 \mathrm{~mm}$, and when combined with in situ subsidence data, $1.25 \mathrm{~m}$ was taken as the construction distance of the HPJG pile group. The construction procedure is illustrated in Figure 10.

According to the parameters calculated based on the genetic algorithm and FOA, the lifting process of HPJG at measurement point $\mathrm{O}$ was predicted (Figure 10). The predicted results and monitored results are listed in Table 4.

The comparison is also shown in Figure 11.

The theoretical error between the monitored data of corner point $\mathrm{O}$ in block $\mathrm{E}$ (Table 4) and the predicted values using the FOA is from 0 to $8.98 \%$, while the theoretical error between the monitored data in Table 3 and the predicted values using the genetic algorithm was from 0.0476 to 0.1075 . The average theoretical errors predicted using the two algorithms are from 0.0590 to 0.0701 .

As shown in Figure 4, the raft foundation of the biochemical pond was divided into six blocks by three temperature joints, and we performed prediction and in situ monitoring during the rectification course for the six blocks. Statistically, the mean error of the two algorithms was 0.0442 and 0.0811 . We made statistical analyses on 30 items of prediction data obtained by using FOA and genetic algorithm in 6 blocks. Meanwhile, checkout is also made to determine whether a significant difference between the two kinds of algorithm exists by Student's $t$-test method; the test level is determined as $\alpha=0.05$. According to the test level, as a result of the computed test statistic $t=1.62, t_{0.05 / 2,29}=2.045, t<t_{0.05 / 2,29}$, and $P>0.05$. We conclude that the difference between the two kinds of algorithms has no statistical meaning; thus, we consider that the prediction result of the two kinds of algorithms has no significant difference. The prediction results of the 


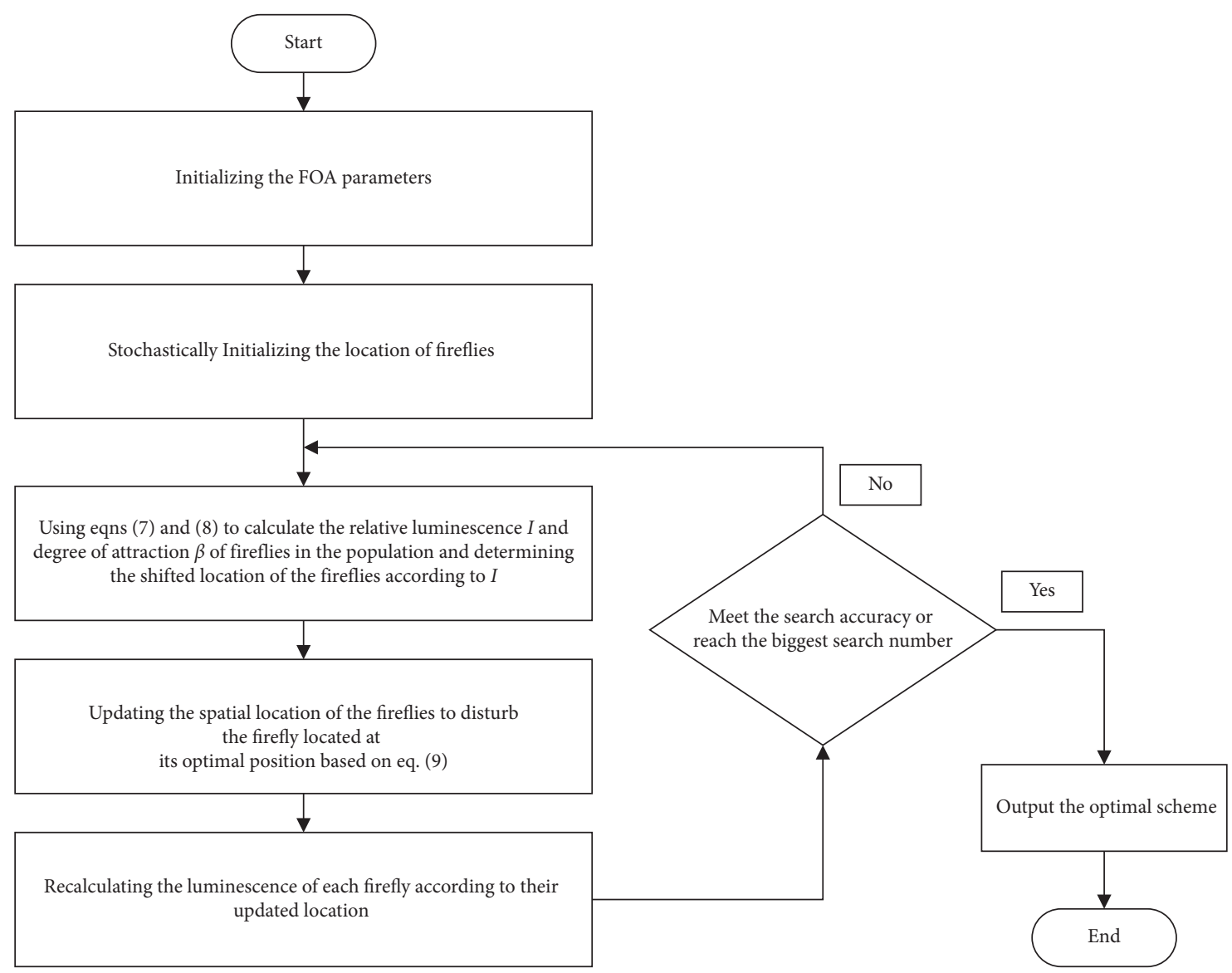

Figure 9: Flow chart for the FOA.

Table 3: Optimized FOA results.

\begin{tabular}{|c|c|c|}
\hline \multicolumn{2}{|c|}{ Parameter } & Goal function \\
\hline $\tan ^{2} \beta$ & $\Delta a$ & $F(x)=\left|W-W_{0}\right|$ \\
\hline 0.4534 & 0.2577 & 0.0079 \\
\hline 0.5312 & 0.263 & 0.0076 \\
\hline 0.4333 & 0.2459 & 0.0067 \\
\hline 0.4102 & 0.2382 & 0.0061 \\
\hline 0.4111 & 0.2137 & 0.0037 \\
\hline 0.3934 & 0.077 & 0.0028 \\
\hline 0.464 & 0.1083 & 0.0021 \\
\hline 0.5389 & 0.2026 & 0.002 \\
\hline 0.5428 & 0.201 & 0.0019 \\
\hline 0.365 & 0.1881 & 0.0019 \\
\hline 0.4083 & 0.1874 & 0.0016 \\
\hline 0.4529 & 0.1825 & 0.0011 \\
\hline 0.4121 & 0.1678 & 0.0004 \\
\hline 0.4131 & 0.1654 & 0.0002 \\
\hline 0.5179 & 0.1667 & 0 \\
\hline
\end{tabular}

two kinds of algorithms meet the requirement of prediction in advance on the uplift height by the following HPJG construction.

5.2. Comparison Analyses of the Algorithms. The FOA obtains the parameter result which entirely meets the optimal target

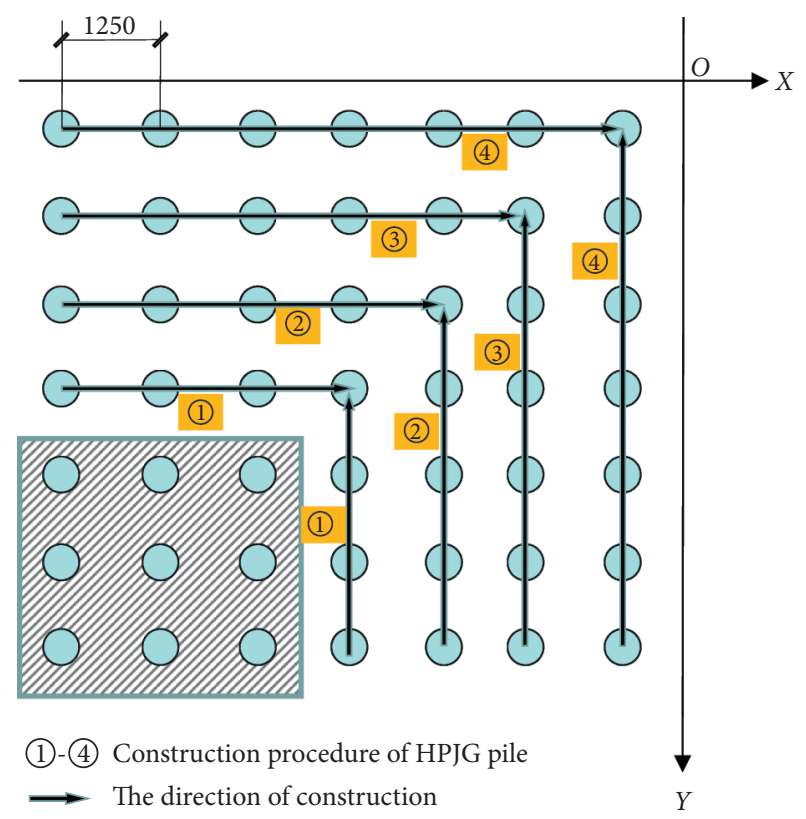

Figure 10: Construction procedure of HPJG pile.

function in the course of optimal parameter search. Furthermore, the FOA has an excellent global optimization function to get high precision with fewer iterations than other 
TABle 4: Optimized FOA results.

\begin{tabular}{lccccc}
\hline Date & $\begin{array}{c}\text { In situ monitoring } \\
\text { data }\end{array}$ & $\begin{array}{c}\text { FOA } \\
\text { Theoretically } \\
\text { predicted values }\end{array}$ & Theoretical error (\%) & Theoretically predicted values & Theoretical error (\%) \\
\hline $2016 / 2 / 22$ & 0 & 0 & 0 & 0 & 0 \\
$2016 / 2 / 26$ & 3.3 & 3.3 & 0 & 3.6 & 9.0909 \\
$2016 / 2 / 28$ & 8.4 & 7.8 & 7.1429 & 8.8 & 7.7619 \\
$2016 / 3 / 2$ & 18.7 & 17.1 & 8.5561 & 17.3 & 7.4866 \\
$2016 / 3 / 4$ & 43.6 & 41.5 & 4.8165 & 62.3 & 2.9817 \\
$2016 / 3 / 7$ & 67.9 & 61.8 & 8.9838 & 60.6 & 10.7511 \\
\hline
\end{tabular}

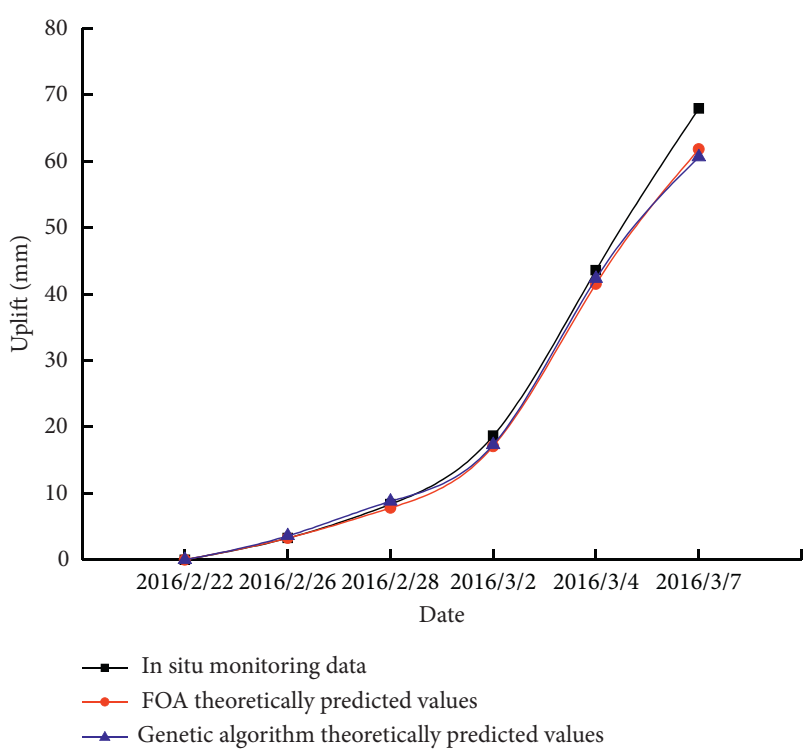

FIGURE 11: Comparison of the predicted uplift effect of HPJG and in situ monitoring data.

algorithms. Thus, the FOA is more effective in searching for the optimal solution according to the target function. This fully shows the feasibility and effectiveness of the FOA in the course of model parameter search in continuous space. Moreover, the comparison of prediction results analyses with in situ monitoring data also shows the applicability of FOA in the uplift prediction model due to HPJG.

From the view of algorithm comparison analyses, the theoretical errors of the two algorithms meet the requirement for the prediction in advance. From the view of complexity, the FOA has two inner loops throughout population $n$ and one outer loop with $t$ iteration times; thus, the FOA has a complexity of $\mathrm{O}\left(n^{2} t\right)$, while the genetic algorithm has a complexity of $\mathrm{O}\left(n^{2}\right)$. The FOA has more complexity with a longer runtime in one loop (approximately $30 \mathrm{~min}$ ) than the genetic algorithm (approximately $20 \mathrm{~min}$ ). However, we can obtain the optimal result of the target function by the FOA in one running time; namely, the running times of the FOA to obtain better precision are far less than those of the genetic algorithm. Moreover, the precision of the optimal prediction from the FOA is better than that from the genetic algorithm. Therefore, the FOA has a greater advantage in terms of effectiveness and precision than the genetic algorithm.

\section{Conclusion and Discussion}

6.1. Conclusion. Using stochastic medium theory to establish the deformation prediction model to predict the uplift due to HPJG, we proposed a new method to make an optimization algorithm through the target function established by the main influence angle $\beta$ and enlarged radius $\Delta \alpha$ searched by the FOA and a genetic algorithm combined with in situ monitoring data. The results show that FOA has a greater advantage in the effectiveness and prediction precision. Statistically, the prediction results can effectively predict the uplift deformation of the proposed building/structure to be reinforced due to multi-HPJG.

Combined with in situ monitoring data and the foundation type of the proposed building/structure to be reinforced, the grouting point space and construction sequence of HPJG are determined by the optimization algorithm according to the prediction results and previous predictions for uplift are quantificationally made in the medium term to avoid secondary damage to the building/structure, which meets the dynamic design concept of geotechnical engineering.

6.2. Discussion. In the present study, we propose a prediction method based on the FOA for uplift due to HPJG. Under the circumstance of even strata and loads, the prediction results through in situ statistics meet the precision requirement. Nevertheless, for complicated strata and load situations, further study is needed that considers the variable factor affecting the uplift parameters under different circumstances to establish a general appraisal and prediction system to enhance prediction effectiveness and precision to meet the requirements of different projects.

\section{Data Availability}

The data used to support this research article are available from the first author on request.

\section{Conflicts of Interest}

The authors declare that there are no conflicts of interest regarding the publication of this paper. 


\section{Acknowledgments}

The authors are grateful to the National Natural Science Foundation of China (Grant no. 51474048) for its financial support, which allowed us to perform this project.

\section{References}

[1] A. Johnson, "Jet grouting aids drive age in loose rock," Tunnels and Tunneling, vol. 10, pp. 53-54, 1989.

[2] D. A. Lewis and G. T. Martin, "North airfield drainage improvement at Chicago-O'hare international airport: soil stabilization using jet grouting," in Proceedings of the Third International Conference on Grouting and Ground Treatment, New Orleans, LA, USA, February 2003.

[3] A. Pinto, R. Tomásio, and G. Marques, "Ground improvement with jet grouting solutions at the new cruise terminal in Lisbon, Portugal," Procedia Engineering, vol. 143, pp. 14951502, 2016.

[4] K. Mussgeer and J. Koinig, "Jet grouting in combination with NATM," Proceedings Rapid Excavation and Tunneling Conference, vol. 1, pp. 292-308, 1987.

[5] E. D. Graf, "Compaction grouting technique and observations," Journal of the Soil Mechanics and Foundations Division, vol. 95, no. 5, pp. 1151-1158, 1969.

[6] E. D. Graf, "Grouting soil improvement and geosynthetics Geotech," Compaction Grouting Technique, vol. 2, pp. 25-28, 1992.

[7] D. Greenwood, "Underpinning by grouting," Ground Engineering (London), vol. 20, pp. 20-32, 1987.

[8] J. Gollegger, Numerical and Analytical Studies of the Effects of Compensation Grouting, Graz University of Technology, Graz, Austria, 2001.

[9] D. R. Brown, "Compaction grouting," Journal of the Soil Mechanics and Foundations Division, ASCE, vol. 99, pp. 589-601, 1973.

[10] M. E. Adel, E. M. Mostafa, and M. B. Ismail, "Model of compaction grouting," Journal of Geotechnical and Geoenvironmental Engineering, vol. 127, no. 11, pp. 955-964, 2001.

[11] W. Compaction, "Grouting-the first thirty years," in Proceedings of the Sepcialty Conference on Grouting in Geotechnical Engineering, pp. 694-707, New Orleans, LA, USA, February 1982.

[12] J. Littlejohn, "The development of practice in permeation and compensation grouting: a historical review (1802-2002)," in Proceedings of the Third International Conference on Grouting and Ground Treatment, pp. 100-144, New Orleans, LA, USA, February 2003.

[13] J. Litwiniszyn, "Fundamental principles of the mechanics of stochastic medium," in Proceedings of the 3rd Conference on Theoretical Applied Mechanics, pp. 18-26, Kharagpur, India, December 1957.

[14] J. Litwiniszyn, "The theories and model research of movements of ground masses," in Proceedings of the European Congress on Ground Movement, pp. 202-209, University of Leeds, Leeds, UK, April 1957.

[15] J. Litwiniszyn, "Effect of porosity on the phenomenon of rock and gas mass outbursts," International Journal of Rock Mechanics and Mining Sciences \& Geomechanics Abstracts, vol. 26, no. 1, pp. 3-15, 1981.

[16] H. C. Shi, M. L. Peng, and C. B. Liu, "Influence of shallow tunnel excavation on ground surface buildings," Chinese
Journal of Rock Mechanics and Engineering, vol. 23, pp. 3310-3316, 2004.

[17] D. I. Harris, R. J. Mair, and J. P. Love, "Compensation grouting to control tilt of big ben clock tower," in Proceedings of the Geotechnical Aspects of Underground Construction in Soft Ground, pp. 225-232, Balkema, Netherlands, November 1999.

[18] W. Z. Tang, G. C. Zhao, and L. D. Zhang, "Design of displacement grouting and prediction of the effect for construction of underground structures," China Civil Engineering Journal, vol. 40, pp. 79-84, 2007.

[19] Y. C. Wang, H. W. Jing, H. J. Su, and J. G. Xie, "Effect of a fault fracture zone on the stability of tunnel-surrounding rock," International Journal of Geomechanics, vol. 17, no. 6, pp. 1-20, 2017.

[20] C. González and C. Sagaseta, "Patterns of soil deformations around tunnels. application to the extension of madrid metro," Computers and Geotechnics, vol. 28, no. 6-7, pp. 445-468, 2001.

[21] C. Sagaseta, "Analysis of undraind soil deformation due to ground loss," Géotechnique, vol. 37, no. 3, pp. 301-320, 1987.

[22] S. K. Au, K. Soga, and M. D. Bolton, "Effect of multiple injection on long-term compensation grouting," Laboratory and Numerical Studies, vol. 2, pp. 657-661, 2002.

[23] A. Contini, A. Cividini, and G. Gioda, "Numerical evaluation of the surface displacements due to soil grouting and to tunnel excavation," International Journal of Geomechanics, vol. 7, no. 3, pp. 217-226, 2007.

[24] L. X. Li, W. Q. Tong, and J. F. Zou, "Study on grouting uplift displacement prediction using stochastic medium theory," Journal of Railway Science and Engineering, vol. 10, pp. 47-51, 2013.

[25] N. Li, P. Zhang, and W. J. Yan, "Numerical modeling of grouting in geoengineering," Journal of Rock Mechanics and Engineering, vol. 21, pp. 326-330, 2002.

[26] D. I. Harris, R. J. Mair, J. P. Love, R. N. Taylor, and T. O. Henderson, "Observations of ground and structure movements for compensation grouting during tunnel construction at Waterloo Station," Géotechnique, vol. 44, no. 4, pp. 691-713, 1994.

[27] Y. Zhang, X.-F. Song, and D.-W. Gong, “A return-cost-based binary firefly algorithm for feature selection," Information Sciences, vol. 419, pp. 561-574, 2017.

[28] Y. Zhang, D.-W. Gong, X.-Y. Sun, and N. Geng, "Adaptive bare-bones particle swarm optimization algorithm and its convergence analysis," Soft Computing, vol. 18, no. 7, pp. 1337-1352, 2014.

[29] Y. Zhang, S. Cheng, Y. Shi, D.-W. Gong, and X. Zhao, "Costsensitive feature selection using two-archive multi-objective artificial bee colony algorithm," Expert Systems with Applications, vol. 137, pp. 46-58, Dec. 2019.

[30] Z. W. Geem, X. S. Yang, and C. L. Tseng, "Harmony search and nature-inspired algorithms for engineering optimization," Journal of Applied Mathematics, vol. 2013, Article ID 438158, 2013.

[31] E. Rashedi, H. Nezamabadi-pour, and S. Saryazdi, "GSA: a gravitational search algorithm," Information Sciences, vol. 213, pp. 267-289, 2010.

[32] K. Gao, Z. Cao, L. Zhang, Z. Chen, Y. Han, and Q. Pan, “A review on swarm intelligence and evolutionary algorithms for solving flexible job shop scheduling problems," IEEE/CAA Journal of Automatica Sinica, vol. 6, no. 4, pp. 904-916, 2019.

[33] H. Yuan, J. Bi, and M. Zhou, "Spatiotemporal task scheduling for heterogeneous delay-tolerant applications in distributed 
green data centers," IEEE Transactions on Automation Science and Engineering, vol. 16, no. 4, pp. 1686-1697, 2019.

[34] W. Deng, J. Xu, Y. Song, and H. Zhao, "An effective improved co-evolution ant colony optimization algorithm multistrategies and its application," International Journal of BioInspired Computing, vol. 16, no. 3, pp. 1-10, 2020.

[35] S. Song, Q. Wang, J. Chen, Y. Li, W. Zhang, and Y. Ruan, "Fuzzy C-means clustering analysis based on quantum particle swarm optimization algorithm for the grouping of rock discontinuity sets," KSCE Journal of Civil Engineering, vol. 21, no. 4, pp. 1115-1122, 2017.

[36] H. Yuan, J. Bi, and M. Zhou, "Multiqueue scheduling of heterogeneous tasks with bounded response time in hybrid green IAAS clouds," IEEE Transactions on Industrial Informatics, vol. 15, no. 10, pp. 5404-5412, 2019.

[37] M. Farooq, "Genetic algorithm technique in hybrid intelligent systems for pattern recognition," International Journal of Innovative Research in Science, Engineering and Technology, vol. 4, no. 4, pp. 1891-1898, 2015.

[38] D. Goldberg, Genetic Algorithms in Search, Optimization and Machine Learning, Addison-Wesley Pub. Co., New York, NY, USA, 1989.

[39] P. C. Liu and M. C. Ye, "Novel bioinspired swarm intelligence optimization algorithm: firefly algorithm," Application Research of Computers, vol. 28, pp. 3295-3297, 2011.

[40] X. S. Yang, "Firefly algorithm, stochastic test functions and design optimisation," International Journal of Bio-Inspired Computation, vol. 2, no. 2, pp. 78-84, 2010.

[41] M.-H. Horng, "Vector quantization using the firefly algorithm for image compression," Expert Systems with Applications, vol. 39, no. 1, pp. 1078-1091, 2012.

[42] X. S. Yang and X. He, "Firefly algorithm: recent advances and applications," International Journal of Swarm Intelligence, vol. 1, no. 1, pp. 36-50, 2013.

[43] K. Jagatheesan, B. Anand, S. Samanta, N. Dey, A. S. Ashour, and V. E. Balas, "Design of a proportional-integral-derivative controller for an automatic generation control of multi-area power thermal systems using firefly algorithm," IEEE/CAA Journal of Automatica Sinica, vol. 6, no. 2, pp. 503-515, 2019. 